\title{
Differential evolution based special protection and control scheme for contingency monitoring of transmission line overloading
}

\begin{abstract}
In designing System Protection Schemes (SPSs) in a power system, protecting transmission lines against severe undesired conditions becomes a serious effort in minimizing line overloading. In this paper, a Special Protection and Control Scheme (SPCS) based on Differential Evolution (DE) algorithm for optimal generation rescheduling to mitigate line overloads in contingency conditions has been presented. Simulation results for various N i 1 contingency conditions within the considered power system under base case load are proposed and validated with those results from the Genetic Algorithm (GA). Minimum severity index has been undertaken as the objective function of this research work. The final results prove that the DE based algorithm gives better than GA in terms of the speed of convergence and minimum generation fuel cost. IEEE 30-bus test system was used to verify the effectiveness of the implemented methods.
\end{abstract}

Keyword: Special protection scheme; Transmission line overloading; Line contingency; Generation rescheduling; Differential evolution (DE) algorithm 